\title{
Propriedades psicométricas da escala de percepção de estressores ocupacionais dos professores (EPEOP)
}

\author{
Silvia Fernandes do Vale \\ Universidade de Fortaleza - Fortaleza - CE \\ Regina Heloisa Maciel \\ Universidade de Fortaleza - Fortaleza - CE \\ Mary Sandra Carlotto \\ Pontifica Universidade Católica do Rio Grande do Sul - Porto Alegre - RS
}

\section{Resumo}

Este estudo investigou as propriedades psicométricas da Escala de Percepção de Estressores Ocupacionais dos Professores - EPEOP. A escala, composta por 22 itens, foi aplicada coletivamente em 404 professores do Ensino Básico de escolas estaduais e municipais de Mossoró (RN), sendo $73,8 \%$ mulheres, com idades entre 20 a 68 . A fidedignidade do instrumento foi alta com alpha de Cronbach de 0,94 . A análise fatorial dividiu a escala em quatro fatores com os seguintes índices de confiabilidade: carga de trabalho $(\alpha=0,88)$, aspectos físicos e ambientais do trabalho $(\alpha=0,88)$, crescimento na carreira e capacitação profissional $(\alpha=0,82)$ e relações com alunos e responsáveis $(\alpha=0,79)$. A média geral da EPEOP foi 46,80 pontos. O instrumento apresentou características psicométricas adequadas para uso em professores, podendo orientar medidas que visem melhoria na qualidade de vida desses profissionais.

Palavras-chave: Escalas; stress ocupacional; trabalho docente.

\section{Psychometric properties of a scale for measuring the perception of occupational stressors by teachers (EPEOP)}

\begin{abstract}
This study investigated the psychometric properties of a scale for measuring the Perception of Occupational Stressors by Teachers (EPEOP). The scale was applied in a sample of 404 teachers of public schools (state or municipal) of basic education of Mossoró (RN), Brazil. $73.8 \%$ were women between 20 to 68 years old. The EPEOP, composed of 22 items, was applied collectively in the participants' schools. The scale reliability was considered high with a Cronbach's alpha of 0.94 . The factorial analysis divided the scale into four factors with the following indices: workload $(\alpha=0.88)$, physical and environmental aspects of work $(\alpha=0.88)$, career growth and professional training $(\alpha=0.82)$, and relationships with students and guardians $(\alpha=0.79)$. The EPEOP overall average for the sample was 46.80 points. The instrument presents adequate psychometric characteristics for evaluating teachers' stress and can orient actions aimed at improving the quality of life of these professionals.
\end{abstract}

Keywords: Scaling; occupational stress; teaching work.

\section{Propiedades psicométricas de la escala de percepción de estresores laborales de maestros (EPEOP)}

\section{Resumen}

Este estudio investigó las propiedades psicométricas de la escala de Percepción de Estresores Laborales de Maestros (EPEOP). La escala fue aplicada en 404 maestros de educación básica de las escuelas del estado y municipales de Mossoró (RN), Brasil. 73,8\% eran mujeres con edades comprendidas entre 20 y 68 años. La escala, composta por 22 ítems, fue aplicada colectivamente en las escuelas de los maestros. La confiabilidad del instrumento fue alta, con alfa de Cronbach de 0.94. La análisis factorial dividió la escala en cuatro factores con los siguientes índices: carga de trabajo $(\alpha=0,88)$, aspectos físicos y ambientales de trabajo $(\alpha=0,88)$, crecimiento profesional y formación profesional $(\alpha=0,82)$ y relaciones con los estudiantes y responsables $(\alpha=0,79)$. El promedio general de la EPEOP fue 46,80 puntos. El instrumento tiene características psicométricas adecuadas para su utilización en los profesores y puede subsidiar medidas para mejorar la calidad de vida de los profesionales. Palabras clave: Escalas; estres ocupacional; trabajo docente. 


\section{Introdução}

Nas últimas décadas, foram desenvolvidas várias pesquisas sobre estresse, associando-o a diferentes variáveis. Dentre essas pesquisas, tem-se observado a preocupação com investigações sobre a relação estresse e trabalho, destacando-se o construto do estresse ocupacional (Lipp, \& Malagris, 2001).

A Organização Internacional do Trabalho (ILO, 2012) aponta que o estresse ocupacional é, na atualidade, uma das mais importantes questões de saúde mundial e tem sido alvo de preocupação em muitos países nos mais diferentes contextos de trabalho. Tal preocupação deve-se ao impacto negativo que o estresse causa na saúde física e mental dos trabalhadores, elevando os já altos índices de afastamento laboral, com evidente diminuição da produtividade nas organizações de trabalho. Cerca de um terço dos trabalhadores tem relatado altos níveis de estresse, estando estes associados a um aumento substancial na utilização dos serviços de saúde (Niosh, 2014).

Os estressores ocupacionais geram reações negativas, causando impactos na organização, uma vez que esta tem que lidar com os prejuízos em virtude do alto índice de absenteísmo do trabalhador, atrasos, erros decorrentes de descuido, acidentes de trabalho, rotatividade do pessoal, como também podem afetar outras áreas da vida do trabalhador, influenciando sua autoestima e causando baixa imunidade, entre outras (Oliveira, 2005).

Os efeitos do estresse ocupacional sobre a saúde do trabalhador em um ambiente laboral em constante e intensa modificação ainda necessitam ser melhor conhecidos e devem fazer parte da agenda de saúde pública (Macedo, \& cols., 2007).

O termo estresse no trabalho ou estresse ocupacional refere-se a um complexo estado físico-psíquico proveniente das exigências e inadequações dos fatores ambientais, organizacionais e humanos no ambiente de trabalho (Bittar, \& cols., 2004). Por isso, depende da maneira como a pessoa lida com a relação entre suas capacidades, habilidades e atributos e os aspectos de seu ambiente de trabalho (Bartlett, 1998).

Trinxet e Cvitanic (2003) definem o estresse ocupacional como um processo no qual o indivíduo, ao perceber as exigências do trabalho como estressoras, passa a ter reações negativas a ele, visto que elas excedem suas habilidades de enfrentamento. De modo semelhante, Paschoal e Tamayo (2004) conceitualizam o estresse ocupacional como um processo estressor-resposta, que envolve desde os fatores de trabalho que excedem a capacidade de enfrentamento do indivíduo (estressores organizacionais) quanto reações fisiológicas, psicológicas e comportamentais aos eventos avaliados como estressores. Trata-se, portanto, de um processo em que o indivíduo percebe as demandas do trabalho como estressoras, as quais, ao exceder sua habilidade de enfrentamento, provocam-lhe reações negativas; esta definição respalda este estudo.
Fatores psicossociais (relacionamento interpessoal, autonomia, conflito e ambiguidade de papéis e fatores relacionados ao desenvolvimento de carreira) apontam como preditores do estresse ocupacional: a natureza da tarefa e do papel ocupacional (estressores relacionados à sobrecarga de trabalho, relacionamentos interpessoais, autonomia, controle e desenvolvimento da carreira); características pessoais (autoestima, comportamento tipo $\mathrm{A}$ ); e as variáveis de natureza situacional (que se referem ao suporte social, conflito entre papéis e ambiguidade do papel) (Canova, \& Porto, 2010; Paschoal, \& Tamayo, 2004). Assim, o estresse ocupacional advém, principalmente, de uma variedade de fontes que estão relacionadas às condições de trabalho em que o trabalhador desenvolve suas atividades laborais, isto é, fatores ambientais e características do trabalho. No entanto, fatores pessoais também são considerados importantes na gênese do estresse (Murta, \& Tróccoli 2009; Paschoal, \& Tamayo, 2004). Na teoria do enfrentamento (coping) (Lazarus, 1993), a percepção do trabalhador de seus recursos frente às demandas e suas habilidades para enfrentá-las são determinantes no aparecimento do estresse (Lipp, \& Malagris, 2001).

Estressores ocupacionais apresentam variabilidade e flutuações de curto prazo (Ohly, Sonnentag, Niessen, \& Zapf, 2010) e especificidades de acordo com a categoria profissional (Laranjeira, 2009). Entre os bancários, Koltermann, Tomasi, Horta e Koltermann (2011) descreveram como maiores fontes estressoras a pressão ou cobrança da chefia, exigência de metas, possibilidade de ser responsabilizado por perdas de valores, insuficiência de pessoal, preocupação com a avaliação do desempenho e ascensão. Em trabalhadores da saúde mental, Santos e Cardoso (2010) identificaram como estressores as condições de trabalho e os relacionamentos no trabalho.

No caso dos agentes de trânsito de Minas Gerais, apresentaram-se como fatores estressores: a incerteza, a falta de controle, a diversidade nas tarefas, os problemas com relação ao conteúdo de trabalho, a falta de feedback, a ausência de perspectivas e progressão na carreira, a falta de participação na tomada de decisões entre outras (Sampaio, Carvalho-Freitas, \& Kemp, 2010). Kilimnik, Bicalho, Oliveira e Mucci (2012) encontraram como fontes estressoras em médicos que atuam em Unidades de Pronto Atendimento as pressões oriundas da relação médico-paciente, sobrecarga de trabalho em virtude da superlotação de pacientes, desgaste físico, o trabalho em equipe e a estrutura física limitada.

No atual contexto de expressivas mudanças no cenário educacional, tanto em termos sociais, tecnológicos e pedagógicos como nas políticas educacionais, observa-se um aumento na quantidade e intensidade de estressores ocupacionais, o que têm gerado reflexos importantes para a saúde do professor. Ensinar é uma atividade, em geral, altamente estressante, com repercussões evidentes na saúde física, mental e no desempenho profissional dos professores (McCarthy, Lambert, O’Donnell, \& Melendres, 2009; Reis, Araújo, Carvalho, Barbalho, \& Silva, 2006). 
Exige-se dos docentes a qualidade de ensino, entretanto, suas condições de trabalho são marcadas pela precarização dos recursos humanos e dos recursos materiais, baixos salários e pelas inúmeras funções que devem exercer dentro do contexto escolar, cenário que contribui para desencadear esgotamento (Gomes, \& Brito, 2006). A presença de diversos fatores ligados ao trabalho dos professores como a falta de interesse e a indisciplina dos alunos (Dalagasperina, \& Monteiro, 2014; Gomes, Montenegro, Peixoto, \& Peixoto, 2010), a falta de estrutura, a falta de diálogo, o autoritarismo da coordenação e da direção da escolar, o individualismo por parte de colegas, os baixos salários (Collie, Shapka, \& Perry, 2012; Dolton, \& Marcenaro-Gutierrez, 2011; Costa, \& Rocha, 2013; Donatelli, \& Oliveira, 2010); a falta de recursos, a falta de tempo, os compromissos excessivos, as turmas de difícil manejo, hostilidade dos pais e a falta de assistência e apoio de administradores e gestores públicos (Khan, 2014); têm sido apontados como estressores. Na atualidade, a profissão docente é comumente reconhecida como tendo elevados níveis de estresse profissional (Hanif, Tariq, \& Nadeem, 2011; Klassen, \& Chiu, 2010).

Diante disso, é necessário dispor de instrumentos que possam caracterizar as fontes geradoras de estresse específicas ao trabalho e contexto docente, tendo em vista que esse indivíduo é um dos atores fundamentais no processo educacional. Tal instrumento pode levar à elaboração de ações voltadas para a prevenção e reabilitação assim como construção de políticas públicas baseadas nesses fatores de risco. No entanto, ainda é escassa no Brasil a construção e validação de instrumentos que possam mensurar os estressores ocupacionais. Paschoal e Tamayo (2004) discutem os instrumentos existentes no Brasil para medir estresse ocupacional ao construírem sua Escala de Estresse no Trabalho (EET). Segundo os autores, havia na época apenas dois instrumentos para realizar esse tipo de medida. Um deles é o QSI (Occupational Stres Indicador), instrumento composto por 167 itens agrupados em 25 fatores que contemplam medidas de estressores, mediadores e consequências físicas e psicológicas. Com relação a esse instrumento, os autores fazem algumas ressalvas, uma vez que a análise fatorial inicial da escala não confirmou os fatores que compõem o instrumento. O segundo instrumento é o SWS Survey (Questionário de estresse, saúde e trabalho), composto por 184 itens fechados, distribuídos em oito escalas. A falta de informações quanto à validação desta última escala dificulta o estabelecimento de conclusões seguras a respeito de sua versão brasileira. AEET é composta por 13 itens e tem como objetivo medir o estresse ocupacional geral. Cada item aborda, ao mesmo tempo, o estressor ocupacional e as variações emocionais. As respostas são colhidas em escala de Likert de 5 pontos. Neto (2007), com o intuito de construir um instrumento que especificasse e avaliasse exclusivamente as fontes de estresse ocupacional em empresas, elaborou a Escala de Estressores Ocupacionais para Trabalhadores em Linha de Produção. O instrumento é composto de 36 itens, divididos em duas fontes de estresse: físico e psicológico.
A fim de compreender as fontes de estresse dos professores, esta pesquisa teve, como objetivo, verificar as propriedades psicométricas de um instrumento de estresse ocupacional de fácil aplicação e que pudesse ser utilizado nos ambientes de trabalho. A escala a ser testada, aqui denominada de Escala de Percepção de Estressores Ocupacionais dos Professores (EPEOP), é específica para professores.

\section{Método}

\section{Participantes}

A amostra, por conveniência, foi composta por 404 professores da educação básica, distribuídos entre a educação infantil ( $n=62 ; 15,4 \%)$, os anos iniciais ( $n=143 ; 35,4 \%)$ e finais $(n=72 ; 17,8 \%)$ do ensino fundamental, a educação de jovens e adultos ( $n=42 ; 10,4 \%)$ e o ensino médio $(n=85 ; 21 \%)$ da rede pública de ensino do município de Mossoró-RN, Brasil. A média de idade da amostra foi de 44,33 anos $(D P=8,37$ anos), variando de 20 a 68 anos, com uma média de tempo de trabalho de 19,91 anos ( $D P=9,03$ anos). As principais características sócio demográficas podem ser vistas na Tabela 1.

Tabela 1. Resumo das características sócio demográficas da amostra.

\begin{tabular}{lcc}
\hline Variáveis & $\boldsymbol{f}$ & $\begin{array}{c}\text { Porcentagem } \\
\text { Válida }\end{array}$ \\
\hline Sexo & & \\
\hline Masculino & 106 & 26,2 \\
Feminino & 298 & 73,8 \\
Não respondeu & 7 & - \\
\hline \multirow{2}{*}{ Idade (anos) } & & \\
\hline$<=36$ & & \\
37 - 44 & 74 & 18,3 \\
$45-53$ & 122 & 30,2 \\
$54+$ & 143 & 35,4 \\
\hline
\end{tabular}

\section{Estado Civil}

\begin{tabular}{lcc} 
Solteiro & 111 & 27,5 \\
Casado & 224 & 55,4 \\
União estável & 6 & 1,5 \\
Divorciado & 37 & 9,2 \\
Viúvo & 26 & 6,4 \\
\hline
\end{tabular}

\section{Escolaridade}

\begin{tabular}{lcc}
\hline Ensino Médio (2o.grau) & 21 & 5,3 \\
Graduação & 145 & 35,9 \\
Especialização & 230 & 56,9 \\
Mestrado & 7 & 1,7 \\
Doutorado & 1 & 0,2 \\
\hline Total & 404 & 100 \\
\hline
\end{tabular}




\section{Instrumentos}

O instrumento foi composto por um questionário sobre dados sociodemográficos e laborais, tais como sexo, idade, estado civil, formação educacional, nível de ensino e tempo de trabalho e da Escala de Percepção de Estressores Ocupacionais dos Professores - EPEOP.

A EPEOP teve como base dois instrumentos. O primeiro foi a Escala de Estressores Ocupacionais das Linhas de Produção, construída e validada por Neto (2007), composta por 36 itens, com o objetivo de avaliar os principais fatores do trabalho (aspectos físicos e psicológicos) que podem ocasionar estresse nos trabalhadores. Em relação a cada um dos 36 itens os sujeitos respondiam com uma nota de 1 a 100 atestando a presença do estressor. Esse tipo de resposta que exige uma nota relacionada a um determinado fator ou índice é comumente utilizada em pesquisas perceptuais (Stevenson, 1960). Na pesquisa de validação da escala original, foram utilizados o Job Content Questionnaire (JCQ) na sua versão em português (vide Araújo, Graça \& Araújo, 2003) e o Inventário de Sintomas de Stress Lipp (ISSL; Lipp, 2000) para verificar a validade de constructo, encontrando-se valores significativos nas correlações $(p<0,01)$. A confiabilidade foi computada por meio de teste-reteste (40 pessoas responderam duas vezes ao teste) e foram encontradas correlações entre 0,98 e 0,99 ( $r$ de Pearson) entre as duas aplicações. Foram utilizados 10 itens dessa escala oriundos de suas duas partes (seis estressores físicos e quatro psicológicos).

O segundo instrumento foi a escala de estresse percebido nas atividades laborais do professor (Carlotto, \& Palazzo, 2006). Com o mesmo objetivo da escala acima (Escala de Estressores Ocupacionais das Linhas de Produção), este instrumento foi desenvolvido pelas autoras para a pesquisa, mas não validado. No entanto, foram verificadas as correlações dos estressores com as dimensões do Maslach Burnout Inventory (MBI) (Maslach \& Jackson, 1986). Os 12 itens que compuseram a escala estudada aqui foram os itens originais propostos pelas autoras, mas modificou-se a forma de resposta. Na pesquisa de Carlotto e Palazzo (2006) as respostas eram dadas em escala Likert de 5 pontos.

Assim, a EPEOP apresenta 22 afirmativas, cada uma abordando um estressor ocupacional, sendo que a resposta ao item indica a reação do respondente a ele. A escala utiliza um sistema de pontuação de 1 a 100 pontos para indicar o quanto o estressor apresentado o afeta negativamente, optando-se, portanto, pelo mesmo tipo de respostas da escala de Neto (2007). O valor "1" corresponde a "não lhe afeta", enquanto "100" representa o valor máximo com que o estressor o afeta negativamente.

\section{Procedimento}

O procedimento de coleta de informações se iniciou após a aprovação pelo Comitê de Ética da Universidade de Fortaleza - UNIFOR (Protocolo $\left.n^{\circ} 111.584 / 2012\right)$. O contato com os professores foi feito por meio de visitas às escolas. Os instrumentos foram aplicados de forma coletiva, em horário de intervalo ou durante reuniões pedagógicas no ambiente escolar (na sala dos professores ou de reuniões). Os participantes foram informados acerca dos objetivos da pesquisa, bem como assegurados do caráter confidencial do estudo e da necessidade de assinar o Termo de Consentimento Livre e Esclarecido. Os dados foram compilados e analisados com o auxílio do pacote estatístico SPSS, versão 21.0.

\section{Resultados}

\section{Análise Fatorial Exploratória}

No teste de Kayser-Meyer-Olkin, a matriz de dados se apresentou como excelente $(\mathrm{KMO}=0,94)$ e o teste de esfericidade de Bartlett foi significativo $\left(\chi^{2}=5189,91\right.$; gl=231; $p<0,001)$. Para verificar a estrutura fatorial em termos de número de fatores foram utilizados os critérios de Kaiser e Cattell (Hayton, Allen, \& Sacarpello, 2004). Com base no primeiro critério (Kaiser), é possível extrair até quatro fatores da EPEOP. O primeiro fator apresentou valor próprio de 10,08 e os demais entre 1,00 e 1,63, explicando conjuntamente $63,27 \%$ da variância total. O critério de Cattell apontou a existência de apenas um fator, como se observa no gráfico de sedimentação (Figura 1).

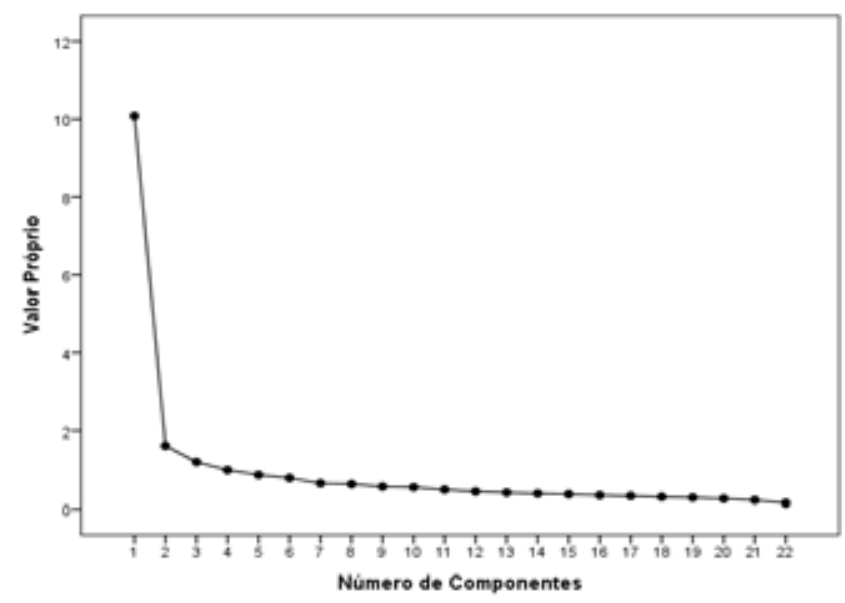

Figura 1. Gráfico de sedimentação (Critério de Cattell)

Em seguida, efetuaram-se múltiplas análises fatoriais exploratórias (AFE) utilizando-se o método dos Componentes Principais $(\mathrm{PC})^{1}$ com rotação por VARIMAX, testando estruturas composta por um, dois e quatro fatores, com base nos resultados do teste de Cattell e do critério de Kaiser. Apesar da hipótese de que os estressores ocupacionais em

1 Foi dada preferência a este método, uma vez que a análise de componentes principais é uma escolha "melhor para fins de redução de dados (traduzindo o espaço das variáveis num espaço ótimo de fatores)." (Figueiredo Filho, \& Silva Junior, 2010, p. 167). 
professores podem ser operacionalizados em estruturas compostas por um ou dois fatores, os resultados da análise sugerem que quatro fatores proporcionam um modelo mais consistente (Tabela 2). O valor de fidedignidade da escala como um todo foi de $\alpha=0,94$ (Alpha de Cronbach).

A solução de quatro fatores mostrou-se mais robusta e simples e com características psicométricas melhores, utilizou-se como critério de inclusão de cada item do questionário nos fatores o valor de corte 0,30 , visto que é um valor usualmente aceitável e que explica pelo menos $9 \%$ da variância total (Nunes, \& Hutz, 2007). Todos os itens carregaram acima do critério nos fatores para os quais foram selecionados (Tabela 2) e os fatores extraídos apresentaram índices de confiabilidade favoráveis.

Tabela 2. Estrutura da Escala de Percepção de Estressores Ocupacionais em Professores $(n=404)$.

\begin{tabular}{|c|c|c|c|c|c|}
\hline \multirow{2}{*}{ Itens } & \multicolumn{4}{|c|}{ Componente } & \multirow{2}{*}{$h^{2}$} \\
\hline & I & II & III & IV & \\
\hline 16. O ritmo do meu trabalho. & 0,73 & 0,19 & 0,21 & 0,21 & 0,66 \\
\hline $\begin{array}{l}\text { 19. A quantidade de atividades que tenho de desenvolver para a escola } \\
\text { fora da sala de aula. }\end{array}$ & 0,72 & 0,09 & 0,29 & 0,16 & 0,64 \\
\hline 17. A execução de atividades burocráticas. & 0,71 & 0,25 & 0,21 & 0,04 & 0,61 \\
\hline 18. A quantidade de atividades que tenho de desenvolver na sala de aula. & 0,65 & 0,25 & 0,33 & 0,14 & 0,63 \\
\hline 08. O fato de desempenhar diferentes papéis na minha profissão. & 0,67 & 0,43 & 0,09 & 0,10 & 0,65 \\
\hline 09. O número de alunos por classe. & 0,57 & 0,24 & 0,04 & 0,36 & 0,52 \\
\hline 10. O número de disciplinas que tenho de lecionar. & 0,51 & 0,30 & 0,25 & 0,11 & 0,43 \\
\hline 04. As condições de limpeza da escola. & 0,16 & 0,78 & 0,15 & 0,14 & 0,69 \\
\hline 02. A iluminação da sala de aula. & 0,21 & 0,77 & 0,11 & 0,16 & 0,68 \\
\hline $\begin{array}{l}\text { 06. Os recursos materiais para que eu desenvolva meu trabalho } \\
\text { satisfatoriamente. }\end{array}$ & 0,20 & 0,70 & 0,31 & 0,18 & 0,67 \\
\hline 05. Os mobiliários da minha sala & 0,12 & 0,72 & 0,20 & 0,31 & 0,67 \\
\hline 03. A ventilação da sala de aula. & 0,37 & 0,63 & 0,09 & 0,13 & 0,56 \\
\hline 21. As possibilidades de crescimento na carreira. & 0,33 & 0,22 & 0,75 & 0,10 & 0,74 \\
\hline 22. As oportunidades de atualização profissional. & 0,35 & 0,19 & 0,74 & 0,09 & 0,73 \\
\hline $\begin{array}{l}\text { 20. O apoio fornecido pela coordenação e colegas para desenvolver meu } \\
\text { trabalho. }\end{array}$ & 0,31 & 0,49 & 0,49 & 0,09 & 0,59 \\
\hline 14. A quantidade de funcionários da escola. & 0,24 & 0,44 & 0,45 & 0,17 & 0,49 \\
\hline 13. As expectativas dos familiares de meus alunos. & 0,14 & 0,26 & 0,47 & 0,64 & 0,72 \\
\hline 12. O relacionamento que tenho com o responsável pelo aluno. & 0,12 & 0,29 & 0,41 & 0,67 & 0,73 \\
\hline 11. O mau comportamento dos alunos. & 0,47 & 0,17 & $-0,05$ & 0,68 & 0,73 \\
\hline 01. O barulho no meu ambiente de trabalho. & 0,29 & 0,48 & $-0,09$ & 0,54 & 0,62 \\
\hline 07. A minha participação nas decisões da escola. & 0,28 & 0,63 & 0,35 & 0,12 & 0,62 \\
\hline 15. O meu salário. & 0,52 & 0,08 & 0,26 & 0,31 & 0,45 \\
\hline Número de Itens & 08 & 06 & 04 & 04 & \\
\hline Valor Próprio & 10,08 & 1,62 & 1,20 & 1,00 & \\
\hline \% Variância Total Explicada & 45,83 & 7,40 & 5,49 & 4,55 & \\
\hline Alfa de Cronbach ( $\alpha)$ & 0,88 & 0,88 & 0,82 & 0,79 & \\
\hline
\end{tabular}

Nota: Carga fatorial considerada satisfatória (acima de 0,30). Fator I = Carga de Trabalho; Fator II = Aspectos Físicos e Ambientais do Trabalho; Fator III = Crescimento na Carreira e Capacitação Profissional; Fator IV = Relações com Alunos e Responsáveis. 


\section{Resultados da escala}

O próximo passo na análise foi calcular as médias obtidas pelos professores na escala e seus fatores. A média geral da EPEOP foi de 46,80 pontos $(D P=19,80)$, variando de 1,27 a 94,55 . Levando em consideração os quatro fatores dos estressores ocupacionais dos professores, o fator "Relações com alunos e responsáveis" apresentou a maior média $(M=50,96 ; D P=22,65)$, seguido do fator "Carga de trabalho" (M=48,88; $D P=21,59)$. Os fatores que apresentaram as menores médias foram "Aspectos físicos e ambientais do trabalho" $(\mathrm{M}=42,85 ; D P=23,34)$ e "Crescimento na carreira e capacitação profissional” ( $M=44,42 ; D P=24,66)$. Os itens que apresentaram maiores médias foram: "o mau comportamento dos alunos" (M=64,06; $D P=29,31)$, seguido de "meu salário" $(M=58,50 ; D P=28,95)$ e os com menores médias foram "o número de disciplinas que tenho de lecionar" (M=36,45; $D P=29,80)$ e "a iluminação da sala de aula" $(\mathrm{M}=37,41 ; \mathrm{DP}=28,25)$.

\section{Discussão}

Discutir os estressores ocupacionais que permeiam o trabalho do professor tem implicações educacionais importantes, visto que os estressores ocupacionais podem ser determinantes do baixo desempenho das atividades do professor, do processo de adoecimento e da desmotivação e, consequentemente, do desempenho dos alunos. Klassen e Chiu (2010) destacam que professores com níveis mais elevados de estresse em sala de aula apresentam níveis baixos de auto eficácia. Explicam Hanif e cols. (2011), que a experiência com estresse no trabalho pode produzir mudanças nas funções psicológicas, fisiológicas e comportamentais do professor.

No entanto, os estudos nesta área são em geral ou qualitativos ou se utilizam de escalas de estressores gerais, não específicos para a categoria dos professores, o que pode causar distorções nos resultados e conclusões encontrados. A escala desenvolvida aqui pode ser utilizada como ferramenta diagnóstica do ambiente de trabalho das instituições educacionais, podendo orientar ações no contexto escolar e, também, políticas públicas que visem a melhoria das condições de trabalho dos professores.

Os resultados apresentados evidenciam que a EPE$\mathrm{OP}$, de maneira geral, é um instrumento satisfatório e específico para o diagnóstico dos estressores ocupacionais da categoria dos professores, com propriedades psicométricas adequadas. Os dados confirmam que os estressores ocupacionais podem ser estruturados a partir de quatro fatores: relações com alunos e responsáveis, carga de trabalho, aspectos físicos e ambientais do trabalho e crescimento na carreira e capacitação profissional.

O fator "Relações com alunos e responsáveis" apresentou a maior média na nossa amostra $(M=50,96$; $D P=$ $22,65)$, ou seja, os professores pontuaram as variáveis que compõem este fator como produtoras de níveis mais elevados de estresse. Dentre elas, destacou-se "o mau comportamento dos alunos" (M=64,06; $D P=29,31)$ como o maior estressor presente neste fator e no trabalho do professor (foi a maior média entre as variáveis da escala como um todo). Carlotto e Palazzo (2006) encontraram que o comportamento inadequado de alunos é um fator de risco para o burnout e um estressor que faz com que os professores apresentem falta de realização profissional, como também preditor da dimensão indolência no Cuestionario para la Evaluación del Síndrome de Quemarse por el Trabajo (CESQT; Questionário de Avaliação do Burnout). Do mesmo modo, Dalagasperina e Monteiro (2014) pontuam a relação professor-aluno como fator de estresse preditor de burnout (indolência). Um estudo com 689 professores portugueses sobre o estresse ocupacional mostrou que dentre as dez principais fontes de estresse mais comuns no trabalho, oito estão relacionadas aos problemas de comportamento inadequado dos alunos que, associados à sensação de incapacidade do professor em manter a disciplina, podem comprometer o bem-estar e eficácia dos docentes (Gomes, \& cols., 2010).

Observou-se que a "Carga de trabalho" ( $M=48,88$; $D P=21,59)$ é o segundo fator de maior impacto no trabalho do professor. O item mais pontuado foi "meu salário" $(\mathrm{M}=$ $58,50 ; D P=28,95)$, também pontuado pelo professor como o segundo maior estressor da escala geral. É importante destacar que o problema econômico em relação aos baixos salários é bastante discutido nas políticas de educação brasileira (Costa, \& Rocha, 2013), visto que os professores não possuem reconhecimento financeiro condizente com sua atividade (Donatelli, \& Oliveira, 2010), impulsionando-o a realizar outras atividades para complementar seu salário, com isso, estende sua carga horária, o que acaba levando, na maioria das vezes, à falta de pausas para descanso, interferindo no bem-estar psicológico. Dolton e Marcenaro-Gutierrez (2011) demonstram a importância do salário do professor no desempenho dos alunos.

Aparecem, com impacto um pouco menor, os dois fatores "Aspectos físicos e ambientais do trabalho" ( $M=42,85$; $D P=23,34)$, e "Crescimento na carreira e capacitação profissional" ( $M=44,42 ; D P=24,66)$. Causa estranheza o fato de os professores da amostra considerarem os aspectos físicos e ambientais do trabalho como fontes de estresse relativamente menos importantes, uma vez que as condições físicas das escolas públicas, principalmente do Norte e Nordeste do país, são bastante precárias (Tavares, Ferreira, \& Maciel, 2008). Por outro lado, a consideração do crescimento na carreira e capacitação profissional como uma fonte de estresse menos importante pode ter relação com a atual política de formação e capacitação de professores que recebe fundos do Governo Federal (Lei n. 11.494, 2007). Isto significa que tal política parece estar tendo um efeito positivo sobre os professores.

O instrumento apresentado mostrou sua sensibilidade na avaliação do impacto dos estressores no contexto do trabalho dos professores, permitindo classificar, em ordem crescente, os fatores geradores de estresse do professor, confirmando a magnitude desse instrumento para futuras pesquisas no campo educacional, orientando medidas que visem a qualidade de vida dos trabalhadores. 
Uma contribuição interessante da escala aplicada é o fato de solicitar respostas com variação de 1 a 100 pontos para o quanto o estressor afeta o indivíduo. Uma variação maior, em comparação as respostas tipo Likert de 5, 7 ou 9 pontos, permite ao respondente uma melhor especificação de sua percepção. Esse tipo de resposta é comumente utilizado em pesquisas perceptuais, após sua primeira utilização por Stevens em 1960 (Stevens, 1960). Ainda não existe consenso quanto ao número de pontos estipulado para escalas, mas sabe-se que a forma de estruturar uma escala de mensuração altera a avaliação psicométrica do respondente (Vieira, \& Dalmoro, 2008). Embora o tipo de resposta solicitada tenha levado a certo estranhamento dos professores, em vista da novidade em relação ao tipo de resposta normalmente solicitada nos questionários, é possível que esse estranhamento tenha sido, em parte, responsável pela melhor especificação da percepção do respondente que resultou no modelo de quatro fatores.

Uma limitação evidente deste estudo foi o fato de a amostra não ter sido probabilística e se ter avaliado somente professores de escolas públicas. Assim, futuras pesquisas com amostras probabilísticas e mais variadas devem ser desenvolvidas, visando o aprimoramento dos resultados sobre o instrumento. Sugere-se, também, estudos que repliquem os resultados obtidos com professores das diferentes regiões do Brasil.

\section{Referências}

Araújo, T. M., Graça, C. C., \& Araújo, E. (2003). Estresse ocupacional e saúde: Contribuições do Modelo Demanda-Controle. Ciência \& Saúde Coletiva, 8(4), 991-1003.

Bartlett, D. (1998). Stress perspectives and processes. Buckingham: Open University Press.

Bittar, A. D. S., Costa, C. C. C., Montini, D., Souza, D. V., Lopes, J., \& Bessa, M. L. (2004). Influência da intervenção ergonômica e o exercício físico no tratamento do estresse ocupacional. Reabilitar, 6(24), 35-44.

Canova, K. R., \& Porto, J. B. (2010). O impacto dos valores organizacionais no estresse ocupacional: Um estudo com professores do ensino médio. Revista de Administração Mackenzie, 11(5), 4-31.

Carlotto, M. S., \& Palazzo, L. S (2006). Síndrome de Burnout e fatores associados: um estudo epidemiológico com professores. Cadernos de Saúde Pública, 22(5), 1017-1026.

Collie, R. J., Shapka, J. D., \& Perry, N. E. (2012). School climate and social-emotional learning: Predicting teacher stress, job satisfaction, and teaching efficacy. Journal of Educational Psychology, 104(4), 189-1204.
Costa, F. R. P., \& Rocha, R. (2013). Resiliência em docentes: Análise das publicações no período de 2000 a 2012. Revista Ciências Humanas, 5(1,2), 141-155.

Dalagasperina, P., \& Monteiro, J. K. (2014). Preditores da síndrome de burnout em docentes do ensino privado. Psico-USF, 19(2), 263-275.

Dolton, P., \& Marcenaro-Gutierrez, O. D. (2011). If you pay peanuts do you get monkeys? A cross-country analysis of teacher pay and pupil performance. Economic Policy, 26(65), 5-55.

Donatelli, S., \& Oliveira, J. A. de (2010). O trabalho de professores da educação básica no Rio Grande do Sul. São Paulo: Fundacentro.

Figueiredo Filho, D. B., \& Silva Júnior, J. A. (2010). Visão além do alcance: Uma introdução à análise fatorial. Opinião Pública, 16(1), 160-185.

Gomes, L., \& Brito, J. (2006). Desafios e possibilidades ao trabalho docente à sua relação com a saúde. Estudos e Pesquisas em Psicologia, 6(1), 49-62.

Gomes, A. R., Montenegro, N., Peixoto, A. M. B. C., \& Peixoto, A. R. B. C. (2010). Stress ocupacional no ensino: um estudo com professores dos $3^{\circ}$ ciclo e ensino secundário. Psicologia \& Sociedade, 22(3), 587-597.

Hanif, R., Tariq, S., \& Nadeem, M. (2011). Personal and job related predictors of teacher stress and job performance among school teachers. Pakistan Journal of Commerce \& Social Sciences, 5(2), 319-329.

Hayton, J. C., Allen, D. G., \& Scarpello, V. (2004). Factor retention decisions in exploratory factor analysis a tutorial on parallel analysis. Organizational Research Methods, 7(2), 191-205

International Labour Office [ILO] (2012). Stress prevention at work checkpoints. Genebra, ILO.

Khan, M. S. (2014). Teacher Burnout: Causes and prevention. Vetri Education, 9(2), 15-22.

Klassen, R. M., \& Chiu, M. M. (2010). Effects on teachers' self-efficacy and job satisfaction: Teacher gender, years of experience, and job stress. Journal of Educational Psychology, 102(3), 741-756.

Kilimnik, Z. M., Bicalho, R. F. S., Oliveira, L. C. V., \& Mucci, C. B. M. R. (2012). Análise do estresse, fatores de pressão do trabalho e comprometimento com a carreira: um estudo com médicos de uma unidade de pronto atendimento de Belo Horizonte, Minas Gerais. Revista Gestão e Planejamento, 12(3), 668-693.

Koltermann, U. T. A. P, Tomasi, E., Horta, B. L., \& Koltermann, A. $P$ (2011). Estresse ocupacional em trabalhadores bancários: prevalência e fatores associados. Revista Saúde, 37(2), 33-48. 
Laranjeira, C. A. (2009). O contexto organizacional e a experiência de estresse: Uma perspectiva integrativa. Revista de Salud Publica, 11(1), 123-33.

Lazarus, R. S. (1993). Coping theory and research: Past, present, and future. Psychosomatic Medicine, 55(3), 234-247.

Lei n. 11.494 de 21 de junho de 2007 (2007). Lei do FUNDEB. Diário Oficial da União - Seção 1, 7. Brasília-DF.

Lipp, M. E. (2000). Manual do inventário de sintomas de stress para adultos de Lipp (ISSL). São Paulo, Casa do Psicólogo.

Lipp, M. E. N., \& Malagris, L. N. (2001). O manejo do estresse. Em B. Rangé (Org.), Psicoterapia comportamental e cognitiva: Transtornos psiquiátricos (pp. 475-490). Campinas: Editorial Psy II.

Macedo, L. E. T. D., Chor, D., Andreozzi, V., Faerstein, E., Werneck, G. L., \& Lopes, C. S. (2007). Estresse no trabalho e interrupção de atividades habituais, por problemas de saúde, no Estudo PróSaúde. Cadernos de Saúde Pública, 23(10), 2327-2336.

Maslach, C., \& Jackson, S. E. (1986). Maslach Burnout Inventory. Palo Alto: Consulting Psychologists Press.

McCarthy, C. J., Lambert, R. G., O'Donnell, M., \& Melendres, L. T. (2009). The relation of elementary teachers' experience, stress, and coping resources to burnout symptoms. The Elementary School Journal, 109(3), 282-300.

Murta, S. G., \& Tróccoli, B. T. (2009). Intervenções psicoeducativas para manejo de estresse ocupacional: Um estudo comparativo. Revista Brasileira de Terapia Comportamental e Cognitiva, 11(1), 25-42.

Neto, R. C. (2007). Construção e validação da escala de estressores ocupacionais das linhas de produção. Tese de Doutorado, Instituto de Psicologia, Pontifícia Universidade Católica de Campinas, Campinas, SP.

National Institute of Occupational Safety and Health [NIOSH] (USA) (2014). Stress at work. Disponível: http://www.cdc.gov/niosh/ docs/99-101/.

Nunes, C. H. S. S., \& Hutz, C. S. (2007). Construção e validação da escala fatorial de socialização no modelo dos cinco grandes fatores de personalidade. Psicologia: Reflexão e Crítica, 20(1), 20-25.
Oliveira, F. A. (2005). Avaliação dos fatores de pressão no trabalho médico e sua relação com o estresse: Um estudo comparativo de casos em clínicas de ultrassonografia da rede privada de Minas Gerais. Dissertação de mestrado em administração, Núcleo de Pós-Graduação e Pesquisa, Centro de Gestão Empreendedora, Belo Horizonte, MG.

Ohly, S., Sonnentag, S., Niessen, C., \& Zapf, D. (2010). Diary studies in organizational research. Journal of Personnel Psychology, 9(2), 79-93.

Paschoal, T., \& Tamayo, A. (2004). Validação da escala de estresse no trabalho. Estudos de Psicologia, 9(1), 45-52.

Reis, E. J. F. B., Araújo, T. M., Carvalho, F. M., Barbalho, L., \& Silva, M. O. (2006). Docência e exaustão emocional. Educação e Sociedade, 27(94), 229-253.

Sampaio, R. F. V., Carvalho-Freitas, M. N., \& Kemp, V. H. (2010). Estressores ocupacionais e qualidade de vida no trabalho de agentes de trânsito. Pesquisas e Prática Psicossociais, 7(1), 3443.

Santos, A. F. O., \& Cardoso, C. L. (2010). Profissionais de saúde mental: estresse e estressores ocupacionais stress e estressores ocupacionais em saúde mental. Psicologia em Estudo, 15(2), 245253.

Stevens, S. S. (1960). Psychophysics of sensory function. American Scientist, 48(2), 226-253. Recuperado: 30 mar. 2014. Disponível: http://www.jstor.org/stable/27827540

Tavares, D.; Ferreira, L. L., \& Maciel, R. H. (2008). O trabalho de professores na educação básica pública no Piauí. São Paulo: FUNDACENTRO.

Trinxet, C. S., \& Cvitanic, V. S. (2003). Control del estrés laboral en los profesores mediante Educación Emocional. Revista de Psicologia de la Universidad de Chile, 12(1), 37-64.

Vieira, K. M., \& Dalmoro, M. (2008). Dilemas na construção de escala tipo likert: $O$ número de itens e a disposição influenciam nos resultados? Em Anais do XXXII Encontro da Associação Nacional de Pós-Graduação em Administração, Rio de Janeiro. Rio de Janeiro: ANPAD. 


\section{Sobre as autoras}

Silvia Fernandes do Vale (sfvale@hotmail.com)

Mestre em Psicologia. Universidade de Fortaleza. Fortaleza, CE.

Regina Heloisa Maciel (reginaheloisamaciel@gmail.com)

Doutora em Psicologia. Universidade de Fortaleza. Fortaleza, CE.

Mary Sandra Carlotto (mscarlotto@gmail.com)

Pós-doutorado em Psicologia. Pontifica Universidade Católica do Rio Grande do Sul. Porto Alegre, RS. 
